\title{
Tables and Maps
}

\section{Tables}

1.1 Ideal-type classification of post-1965 ethnic communities

2.1 Racial and ethnic distribution of residents in

Koreatown, 1990-2000

2.2 Estimated count of organizations within and outside of Koreatown

5.1 Table of select 1.5 and 2nd generation-led organizations in Koreatown, Los Angeles

7.1 Profile of past and present Korean members at KIWA and KYCC, 2000

Maps

2.1 Median household income in Los Angeles County, 2000

2.2 Percent Korean American population in 